\title{
Post-operative atrial fibrillation after off-pump coronary artery bypass grafting
}

\author{
Shamsher Singh Lohchab ${ }^{1}$ (D) Ashwani Kumar ${ }^{2}$
}

Received: 30 May 2019 / Accepted: 3 July 2019 /Published online: 2 August 2019

(C) Indian Association of Cardiovascular-Thoracic Surgeons 2019

Post-operative atrial fibrillation (POAF) after cardiac surgery is a common complication. The incidence of POAF varies from 20 to $60 \%$ as reported in the literature, being $20-30 \%$ in isolated coronary artery bypass surgery (CABG), $35-40 \%$ in isolated valve surgery and $35-60 \%$ in combined $C A B G$ and valve surgery. There is a reported twofold increase in all-cause 30-day and 6-month mortality after POAF. There is higher risk of stroke, reoperation for bleeding, infection, renal or respiratory failure, cardiac arrest, cerebral adverse events and a need for permanent pacemaker implantation in patients who have this complication, thus elevating the cost of hospitalisation [1].

POAF is multifactorial in origin. Causes are further classified as acute factors related to surgical procedure and chronic factors associated with advanced age and structural heart disease. Several proarrhythmogenic mechanisms, inflammation and $\beta$-adrenergic activation play an important role in POAF [2]. Increased susceptibility of POAF has been found in patients with diabetes and metabolic syndrome as a result of decreased mitochondrial function [3]. The Bioenergetic Health Index has recently been used to characterise decreased mitochondrial function following cardiac surgery and it may presage as a predictor of POAF [4].

Pericardial fluid collection and removal of anterior pericardial fat pad have also been attributed in causation [1]. Correction of electrolyte imbalance-hypokalaemia and hypomagnesaemia - is widely practised for the prevention of POAF. However, some studies have shown that potassium and magnesium supplementation does not protect against atri-

Shamsher Singh Lohchab

sslohchab@gmail.com

1 Department of Cardiac Surgery, Pt. B D Sharma PGIMS, Rohtak, Haryana 124001, India

2 Department of Cardiology, Pt. B D Sharma PGIMS, Rohtak, Haryana, India al fibrillation and higher magnesium levels even may increase the risk of POAF [5]. Similarly, milrinone use, which is frequent following cardiac surgery, may lead to POAF [6].

Some studies have shown reduced incidence of atrial fibrillation $(\mathrm{AF})$ in off-pump CABG. Current data, however, do not emphatically answer the question of whether the incidence of post-CABG AF is reduced by off-pump surgery. Decreased incidence of $\mathrm{AF}$ following off-pump $\mathrm{CABG}$ has been attributed to the avoidance of cardiopulmonary bypass-associated inflammatory response and global myocardial ischemia. Ischemia with off-pump CABG is regional, whereas it is global with cardioplegic arrest. The latter may jeopardise supply to the sub-endocardium and interventricular septum and is especially risky in patients with severe left ventricle dysfunction and acute myocardial infarction, as atrial dilatation may occur leading to the risk of AF. Atrial dilatation is the final pathway mechanism of $\mathrm{AF}$, which is less likely to happen in off-pump CABG, as there is less manipulation of the right atrium due to avoidance of cannulation. Less blood loss and decreased pericardial collections after off-pump CABG may be protective against $\mathrm{AF}$, as a result of less direct pressure on the right atrium [7].

For preoperative prophylaxis, current guidelines recommend $\beta$-blockers $24 \mathrm{~h}$ prior to surgery for patients undergoing CABG or who have an ejection fraction greater than $30 \%$. In other patients predisposed to POAF, amiodarone should be administered for 7 days preoperatively. Corticosteroids, although may prevent $\mathrm{AF}$, are not advocated due to the risk of hyperglycaemia, immunosuppression, impaired wound healing and increased gastrointestinal complications. The use of statins, from 4 weeks preoperatively to the evening before surgery, has conflicting data and thus, recommendations for preoperative statin prophylaxis cannot be made at present. Diltiazem is reasonable in high-risk patients who are not taking $\beta$-blockers. Digoxin is not recommended for preoperative prophylaxis. The role of anticoagulants and angiotensin-converting enzyme (ACE) inhibitors is not clear [1] and omega-3 fatty acids, as per OPERA trial 2017, too have no role [8]. 
Intraoperatively, posterior pericardiotomy and preservation of anterior fat pad may significantly reduce the incidence of POAF. Left atrial appendage (LAA) exclusion has emerged as a target for stroke prevention and an alternative to long-term anticoagulation therapy. Quite often there may not be a requirement for the treatment of POAF, as episodes may resolve without intervention. Persistent AF and occurring in haemodynamically unstable patients require management with one or more of the three medications: $\beta$-blockers, amiodarone and calcium channel blockers, usually using a single agent and addition of a second medication as per requirement. Concurrent use of all three may lead to bradycardia and hypotension. Between rate control or rhythm control strategy, neither treatment strategy showed a net clinical advantage over the other. Immediate electrical cardioversion should be done for hemodynamically unstable patients [1].

The prospective study by Ghurram et al. [9], later in this issue of the journal, in 748 patients on POAF in Indian patients undergoing off-pump CABG, is a well-conducted study with appropriately designed statistical analysis and the authors are worthy of congratulations for it. In this study, the overall incidence of POAF was $16.7 \%$, which is lower than that of onpump CABG, an observation reported in other studies too. It is pertinent to mention that in India, a higher percentage of patients undergo off-pump CABG in comparison to the rest of the world. The reduction in the incidence of post-operative AF may add one more point in favour of off-pump CABG.

The study however has certain limitations. AF has been detected only by serial ECGs, whereas guidelines recommend continuous telemetry or Holter monitoring to detect intermittent episodes of AF. Thus, the actual incidence may be higher, reflecting the weakness of this study. The finding of low incidence of POAF as compared with other studies globally, as claimed by the authors, should be viewed factoring the abovementioned fact. The study has pointed out only advanced age and sepsis as risk factors for POAF. Old age is a well-known risk factor for AF. The incidence of sepsis in this study was $11.2 \%$, with a higher incidence of $33.1 \%$ in the POAF group, leading to decreased survival. Sepsis-causing AF due to systemic inflammatory response is a new proposition in this study, as claimed by the authors. Sepsis itself can be the cause of higher morbidity and mortality and POAF may be simply a manifestation of sepsis. On the other hand, POAF can cause sepsis [1] and it is difficult to interpret this proposition like egg and hen controversy. Reduced survival in patients who develop POAF, another finding in the study, is a well-known fact and there is nothing new. Pericardial fluid collection, being highly inflammatory in nature, leads to increased oxidative stress and has been incriminated recently as an important cause of POAF [10], but the study by Ghurram et al. is silent regarding this observation.

Interpretation of this study is further confounded by its being an observational study sans any control group and poorly defined AF monitoring. It is silent on the role of revascularisation and pericardial collection, with conflicting message of sepsis as a cause of POAF. Yet, it is a unique study from Indian subcontinent providing the much-needed data regarding POAF following off-pump CABG. However, more robust data are required about POAF in off-pump CABG to know the incidence, precise mechanisms, risk factors, prophylaxis, treatment and outcome in comparison with conventional on-pump CABG.

\section{References}

1. Greenberg JW, Lancaster TS, Schuessler RB, Melby SJ. Postoperative atrial fibrillation following cardiac surgery: a persistent complication. Eur J Cardiothorac Surg. 2017;52:665-72.

2. Maesen B, Nijs J, Maessen J, Allessie M, Schotten U. Postoperative atrial fibrillation: a maze of mechanisms. Europace. 2012;14:159-74.

3. Montaigne D, Marechal X, Lefebvre P, et al. Mitochondrial dysfunction as an arrhythmogenic substrate: a translational proof-ofconcept study in patients with metabolic syndrome in whom postoperative atrial fibrillation develops. J Am Coll Cardiol. 2013;62: 1466-73.

4. Chacko BK, Kramer PA, Ravi S, et al. The Bioenergetic Health Index: a new concept in mitochondrial translational research. Clin Sci (Lond). 2014;127:367-73.

5. Lancaster TS, Schill MR, Greenberg JW, et al. Potassium and magnesium supplementation do not protect against atrial fibrillation after cardiac operation: a time-matched analysis. Ann Thorac Surg. 2016;102:1181-9.

6. Fleming GA, Murray $\mathrm{KT}, \mathrm{Yu} \mathrm{C}$, et al. Milrinone use is associated with postoperative atrial fibrillation after cardiac surgery. Circulation. 2008;118:1619-25.

7. Athanasiou T, Aziz O, Mangoush O, et al. Does off-pump coronary artery bypass reduce the incidence of post-operative atrial fibrillation? A question revisited. Eur J Cardiothorac Surg. 2004;26:701-10.

8. Ad N, Massimiano PS. Postoperative atrial fibrillation: adding (fish) oil to the fire. J Thorac Cardiovasc Surg. 2018;155:252-3.

9. Ghurram A, Krishna N, Bhaskaran R, Kumaraswamy N, Jayant A, Varma PK. Patients who develop post-operative atrial fibrillation have reduced survival after off-pump coronary artery bypass grafting. Indian J Thorac Cardiovasc Surg. 2019. https://doi.org/ 10.1007/s12055-019-00844-9.

10. Kramer PA, Chacko BK, George DJ, et al. Decreased Bioenergetic Health Index in monocytes isolated from the pericardial fluid and blood of post-operative cardiac surgery patients. Biosci Rep. 2015;35:e0237.

Publisher's note Springer Nature remains neutral with regard to jurisdictional claims in published maps and institutional affiliations. 